\title{
Electromagnetic Sources in Moving Simple Medium
}

\author{
Johannsen, Günther
}

Published in:

Journal of Mathematical Physics

Link to article, DOI:

$10.1063 / 1.1665123$

Publication date:

1970

Document Version

Publisher's PDF, also known as Version of record

Link back to DTU Orbit

Citation (APA):

Johannsen, G. (1970). Electromagnetic Sources in Moving Simple Medium. Journal of Mathematical Physics, 11(11), 3251-3253. https://doi.org/10.1063/1.1665123

\section{General rights}

Copyright and moral rights for the publications made accessible in the public portal are retained by the authors and/or other copyright owners and it is a condition of accessing publications that users recognise and abide by the legal requirements associated with these rights.

- Users may download and print one copy of any publication from the public portal for the purpose of private study or research.

- You may not further distribute the material or use it for any profit-making activity or commercial gain

- You may freely distribute the URL identifying the publication in the public portal

If you believe that this document breaches copyright please contact us providing details, and we will remove access to the work immediately and investigate your claim 


\title{
Electromagnetic Sources in Moving Simple Medium
}

\author{
GÜNTHER JOHANNSEN \\ Danish Engineering Academy, Electrical Department (formally Laboratory of Electromagnetic Field Theory) \\ The Technical University of Denmark, Lyngby, Denmark
}

(Received 4 May 1970)

\begin{abstract}
A retarded potential tensor (4-vector) is derived in an arbitrary system of inertia for an arbitrary electromagnetic source in a moving homogeneous, isotropic, nondispersive, lossless dielectric. The velocity is uniform, and the result is relativistic correct.
\end{abstract}

\section{INTRODUCTION}

The differential equation for the potential tensor is simplest in the system of inertia $K_{m}$ where the medium is at rest. A 4-dimensional integral representation for the potential tensor can readily be obtained by using the 4-dimensional Green's theorem. One may carry out the integration with respect to the time coordinate to get the space integral representation for the (retarded) potential tensor.

As pointed out in Ref. 1, the space integral representation in $K_{m}$ is not very utilizable in another system of inertia $K$ which is in uniform motion relative to $K_{m}$. This is due to the fact that space-time coordinates mix under the Lorentz transformation from $K$ to $K_{m}$.

It is shown that the 4-dimensional integral representation for the potential tensor in $K_{m}$ can be transformed to an arbitrary system of inertia $K$. Integrating with respect to the time coordinate then leads to the space integral representation for the (retarded) potential tensor in $K$. The results found here are in agreement with those in Refs. 2 and 3, where the pertinent differential equation is integrated by using Fourier transformation and an operational method, respectively.

\section{INTEGRAL REPRESENTATIONS OF THE POTENTIAL TENSOR}

We use Cartesian tensor notation. By a tensor we understand a tensor defined on the Lorentz transformation group. Latin subscripts run from 1 to 4 ; Greek subscripts run from 1 to 3 . The coordinate $x_{4}$ is equal to $i c t$, where $t$ is the time and $c$ the speed of light in vacuum; therefore, the metric tensor in 4space is equal to the Kronecker symbol $\delta_{i j}$ (when Cartesian spatial coordinates are used), and we do not distinguish between contravariant and covariant tensors. Repeated subscripts obey the summation convention, and commas in subscripts denote partial differentiation with respect to coordinates (or covariant differentiation, since the metric tensor is independent of the coordinates).

The potential tensor $A_{i}$ satisfies a differential equation, ${ }^{1,4}$ which in an arbitrary system of inertia $K$ can be written as a tensor equation:

$$
\begin{gathered}
A_{i, n n}-\kappa A_{i, s t} U_{s} U_{t}=-S_{i}, \\
\kappa \equiv \frac{n^{2}-1}{c^{2}}, \quad n \equiv \frac{c}{c^{\prime}}, \quad c^{\prime} \equiv \frac{1}{(\mu \epsilon)^{\frac{1}{2}}}, \\
S_{i} \equiv \mu\left(J_{i}+\frac{\kappa}{n^{2}} J_{r} U_{r} U_{i}\right) .
\end{gathered}
$$

$\mu$ and $\epsilon$ are the permeability and the dielectric constant of the medium. $U_{i}$ is the velocity tensor of $K_{m}$, and $J_{i}$ is the current density tensor. ${ }^{1,5}$

Let primed quantities refer to $K_{m}$. Since $U_{i}^{\prime}=$ $(0,0,0, i c)$, we get from (1)

$$
A_{i, n n}^{\prime}+c^{2} \kappa A_{i, 44}^{\prime}=A_{i, v v}^{\prime}+n^{2} A_{i, 44}^{\prime}=-S_{i}^{\prime} .
$$

Introducing new variables by $y_{\rho}^{\prime} \equiv x_{\rho}^{\prime}$ and $y_{4}^{\prime} \equiv$ $n^{-1} x_{4}^{\prime}$, we get from (2)

$$
A_{i, n n}^{\prime}\left(y_{r}^{\prime}\right)=-S_{i}^{\prime}\left(y_{r}^{\prime}\right)
$$

where $\left(y_{r}^{\prime}\right) \equiv\left(y_{1}^{\prime}, y_{2}^{\prime}, y_{3}^{\prime}, y_{4}^{\prime}\right)$.

For the solution of (3) see Ref. 5, p. 146. The result is

$$
A_{i}^{\prime}\left(y_{r}^{\prime}\right)=\left(4 \pi^{2}\right)^{-1} \iiint \int_{-\infty}^{\infty}\left(S_{i}^{\prime}\left(z_{r}^{\prime}\right) / \sum_{r=1}^{4}\left(z_{r}^{\prime}-y_{r}^{\prime}\right)^{2}\right) d V\left(z_{r}^{\prime}\right) .
$$

Substituting back to variables $x_{r}^{\prime}$, we obtain

$$
A_{i}^{\prime}\left(x_{r}^{\prime}\right)=\iiint \int_{-\infty}^{\infty} \int_{i}^{\prime}\left(z_{r}^{\prime}\right) G^{\prime}\left(z_{r}^{\prime}-x_{r}^{\prime}\right) d V\left(z_{r}^{\prime}\right),
$$

where

$$
G^{\prime}\left(z_{r}^{\prime}-x_{r}^{\prime}\right) \equiv\left(4 \pi^{2} n\right)^{-1}\left[u_{r}^{\prime} u_{r}^{\prime}+\left(\kappa / n^{2}\right)\left(u_{r}^{\prime} U_{r}^{\prime}\right)^{2}\right]^{-1}
$$

and

$$
u_{r}^{\prime} \equiv z_{r}^{\prime}-x_{r}^{\prime}
$$

The poles of the function $G^{\prime}$ are given by $u_{4}^{\prime}=$ $\tau_{ \pm}^{\prime} \equiv \pm i n\left(u_{\rho}^{\prime} u_{\rho}^{\prime}\right)^{\frac{1}{2}}$. As in Ref. 5 we can deform the contour for the $z_{4}^{\prime}$ integration to circumvent one of the poles. Since advanced potentials are not of interest, we consider the pole $\tau_{-}^{\prime}$.

From (5) we get

$$
A_{i}^{\prime}\left(x_{r}^{\prime}\right)=\iiint_{-\infty}^{\infty} \oint_{L^{\prime}} S_{i}^{\prime}\left(z_{r}^{\prime}\right) G^{\prime}\left(u_{r}^{\prime}\right) d u_{4}^{\prime} d u_{3}^{\prime} d u_{1}^{\prime} d u_{2}^{\prime},
$$

where $L^{\prime}$ is a closed path surrounding $\tau_{-}^{\prime}$. 
Equation (6) is valid in $K_{m}$; we now transform (6) to an arbitrary system of inertia $K$. Let $x_{r}=a_{r s} x_{s}^{\prime}$ be the proper Lorentz transformation connecting coordinates in $K$ with coordinates in $K_{m}$. Multiplying (6) by $a_{j i}$ and substituting $x_{r}^{\prime}$ by $x_{r}$, we see that the left side in (6) (which is a tensor) becomes $A_{j}\left(x_{r}\right) . a_{j i}$ is independent of the coordinates, and, since $S_{i}^{\prime}$ is a tensor, we have $a_{j i} S_{i}^{\prime}\left(z_{r}^{\prime}\right)=S_{j}\left(z_{r}\right)$ if $z_{r}$ and $z_{r}^{\prime}$ are connected by the proper Lorentz transformation, i.e., $z_{r}=a_{r s} z_{s}^{\prime}$. Furthermore, $G^{\prime}$ transforms like an invariant, i.e.,

$$
G\left(u_{r}\right)=\left(4 \pi^{2} n\right)^{-1}\left[u_{r} u_{r}+\left(\kappa / n^{2}\right)\left(u_{r} U_{r}\right)^{2}\right]^{-1}
$$

Without loss of generality, we choose $a_{r s}$ so that

$$
\begin{array}{ll}
x^{1}=x_{1}^{\prime}, & x_{2}=x_{2}^{\prime}, \\
x_{3}=\gamma\left(x_{3}^{\prime}+i \beta x_{4}^{\prime}\right), & x_{4}=\gamma\left(x_{4}^{\prime}-i \beta x_{3}^{\prime}\right),
\end{array}
$$

where $\gamma \equiv\left(1-\beta^{2}\right)^{-\frac{1}{2}}, \beta \equiv v / c$, and $v$ is the velocity of $K$ relative to $K_{m}$ (Fig. 1).

To see how the poles to be circumvented are transformed under the Lorentz transformation, we consider the tensor equation

$$
u_{r} u_{r}+\left(\kappa / n^{2}\right)\left(u_{r} U_{r}\right)^{2}=0,
$$

which is a 3-dimensional hypersurface in Minkowski 4-space. In $K_{m}(8)$ reduces to $\left(u_{4}^{\prime} / i\right)^{2}-\left(n u_{3}^{\prime}\right)^{2}=\left(n \rho^{\prime}\right)^{2}$ and $\rho^{\prime} \equiv\left(u_{1}^{\prime 2}+u_{2}^{\prime 2}\right)^{\frac{1}{2}}$ which in Euclidean 2-space is the equation of a hyperbola (Cartesian coordinates $\left.u_{4}^{\prime} / i, u_{3}^{\prime}\right)$. In Fig. 2 the situation is illustrated in a way due to Minkowski. ${ }^{6}$

When $n \beta<1$ and $u_{3}$ is given, there is one pole to be circumvented. When $n \beta>1$ (this situation is shown in Fig. 2), there are two poles to be circumvented if $u_{3}>|a|^{\frac{1}{2}} \rho, a \equiv\left[1-(n \beta)^{2}\right] /\left(1-\beta^{2}\right)$, and no poles are to be circumvented if $u_{3}<|a|^{\frac{1}{2}} \rho$ (i.e., $A_{i}$ is equal to zero).

The roots in (8) are given by

$$
\begin{aligned}
\frac{u_{4}}{i}=\tau_{ \pm} & \equiv \frac{n \beta}{1-(n \beta)^{2}} \\
& \times\left[\left(n-n^{-1}\right) u_{3} \pm\left(\beta^{-1}-\beta\right)\left(u_{3}^{2}+a \rho^{2}\right)^{\frac{1}{2}}\right] .
\end{aligned}
$$

Since $n \beta<1$ implies that $a>0$ and $\left(\beta^{-1}-\beta\right)>$ $n-n^{-1}>0$, we actually see that $\tau_{+}>0$ and $\tau_{-}<0$. In this case, from (6), we can deduce

$$
A_{i}\left(x_{r}\right)=\iiint_{-\infty}^{\infty} \oint_{L} S_{i}\left(z_{r}\right) G\left(u_{r}\right) d u_{4} d u_{3} d u_{1} d u_{2},
$$

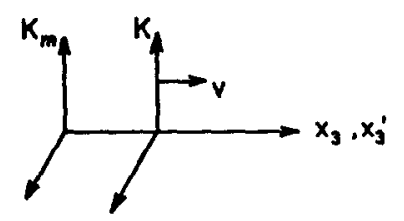

FIG. 1. The motion of $K$ relative to $K_{m}$

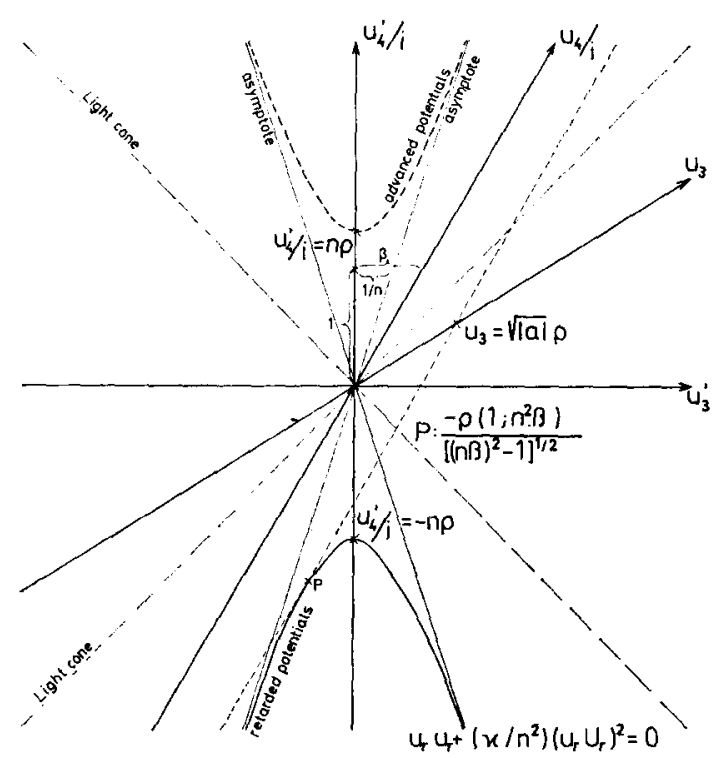

FIG. 2. Location of poles of the function $G$ in Minkowski space.

where $L$ circumvents $\tau_{-}$. By the method of residues, we get

$$
\begin{array}{r}
A_{i}\left(x_{r}\right)=\frac{1}{4 \pi} \iiint_{-\infty}^{\infty} \frac{S_{i}\left(z_{\rho}, x_{4}+\tau_{-}\right)}{\left[\left(z_{3}-x_{3}\right)^{2}+a \rho^{2}\right]^{\frac{1}{2}}} d V\left(z_{\rho}\right), \\
n \beta<1 .
\end{array}
$$

In the case of $n \beta>1$, we actually see from (9) that $\tau_{ \pm}>0$ when $u_{3}<-|a|^{\frac{1}{2}} \rho$, that $\tau_{ \pm}$are imaginary when $-|a|^{\frac{1}{2}} \rho<u_{3}<|a|^{\frac{1}{2}} \rho$, and finally that $\tau_{ \pm}<0$ when $u_{3}>|a|^{\frac{1}{2}} \rho$. From (6) we get

$$
A_{i}\left(x_{r}\right)=\iiint_{-\infty}^{\infty} \oint_{L} S_{i}\left(z_{r}\right) G\left(u_{r}\right) d u_{4} d u_{3} d u_{1} d u_{2},
$$

where the $u_{3}$ integration is to be taken from $\infty$ to $|a|^{\frac{1}{2}} \rho$ ( $L$ surrounding $\left.\tau_{-}\right)$and back to $\infty$ ( $L$ surrounding $\tau_{+}$). It turns out that ${ }^{?}$

$$
\begin{aligned}
A_{i}\left(x_{r}\right)= & -\frac{1}{4 \pi} \iiint_{-\infty}^{\infty} \frac{S_{i}\left(z_{\rho}, x_{4}+\tau_{+}\right)+S_{i}\left(z_{\rho}, x_{4}+\tau_{-}\right)}{\left[\left(z_{3}-x_{3}\right)^{2}+a \rho^{2}\right]^{\frac{1}{2}}} \\
& \times \theta\left[z_{3}-\left(x_{3}+|a|^{\frac{1}{2}} \rho\right)\right] d V\left(z_{\rho}\right)
\end{aligned}
$$$$
n \beta>1 \text {, }
$$

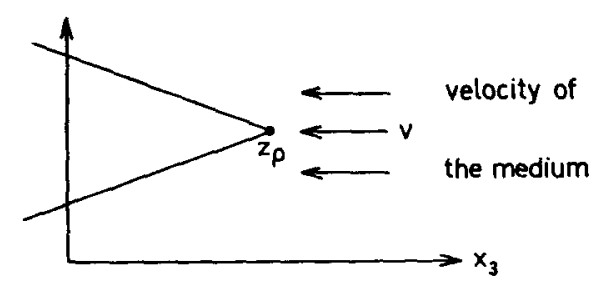

FIG. 3. Conical region in $K$, where a source point contributes to the field, $n \beta>1$. 
where $\theta$ is the unit step function, i.e.,

$$
\begin{aligned}
\theta(t) & =0 \text { when } t<0 \\
& =1 \text { when } t \geq 0 .
\end{aligned}
$$

Equations (11) and (13) hold in an arbitrary system of inertia. The step function in (13) implies that a source point $\left(z_{\rho}\right)$ contributes to the field only at points inside a conical region given by $\left(x_{3}-z_{3}\right) \leq-|a|^{\frac{1}{2}} \rho$.

\section{TIME HARMONIC SOURCES, 3- DIMENSIONAL REPRESENTATION}

Let the source be time harmonic in $K: S_{i}\left(x_{r}\right)=$ $S_{i}\left(x_{\boldsymbol{p}}\right) e^{-k x_{4}}$, where $k \equiv \omega / c$ and $\omega$ is the frequency. Omitting the time factor $e^{-k x_{4}}$, we derive from (9)

$S_{i}\left(z_{\rho}, x_{4}+\tau_{-}\right)$

$$
\begin{aligned}
= & S_{i}\left(z_{\rho}\right) \exp \left[-i k b\left(z_{3}-x_{3}\right)\right] \\
& \times \exp \left\{i k(n / a)\left[\left(z_{3}-x_{3}\right)^{2}+a \rho^{2}\right]^{\frac{1}{2}}\right\},
\end{aligned}
$$

where

$$
b \equiv \frac{n \beta}{1-(n \beta)^{2}}\left(n-\frac{1}{n}\right)=\frac{\beta \kappa c^{2}}{1-(n \beta)^{2}} .
$$

Furthermore, we derive

$$
\begin{aligned}
& S_{i}\left(z_{\rho}, x_{4}+\tau_{-}\right)+S_{i}\left(z_{\rho}, x_{4}+\tau_{+}\right) \\
& \quad=2 S_{i}\left(z_{\rho}\right) e^{-i k b\left(z_{3}-x_{3}\right)} \cos \left\{(k n / a)\left[\left(z_{3}-x_{3}\right)^{2}+a_{\rho}^{2}\right]^{\frac{1}{2}}\right\} .
\end{aligned}
$$

From the definition of $S_{i}$, and making use of the continuity equation $J_{\rho, \rho}=-J_{4,4}=i \omega \rho=k J_{4}$, we get, for the spatial components of $S_{i}$,

$$
\begin{aligned}
S_{\lambda}\left(z_{\rho}\right)=\mu\left(\delta_{\lambda v}+\frac{\kappa}{n^{2}} U_{\lambda} U_{v}\right) & J_{v}\left(z_{\rho}\right) \\
& +i \frac{\mu \kappa c^{2}}{n^{2}} \gamma U_{\lambda} J_{v, v}\left(z_{\rho}\right) .
\end{aligned}
$$

Similarly,

$$
\frac{c}{i} S_{4}\left(z_{\rho}\right)=\frac{\mu c^{2}}{i \omega}\left(1-\frac{\kappa c^{2}}{n^{2}} \gamma^{2}\right) J_{v, v}+\frac{\mu \kappa c^{2}}{n^{2}} \gamma U_{v} J_{v} .
$$

Substituting (14)-(17) into (11) and (13) leads to expressions which are in agreement with Ref. 1.

The field vector $\bar{E}$ may be obtained by using the equation $\bar{E}=-\nabla \Phi+i \omega \bar{A}$ (Ref. 1; it can be shown that $\bar{E}=-\nabla \Phi-\partial \bar{A} / \partial t$ holds in any system of inertia). By some calculation the results may be transformed to an expression as given in Ref. 8.

\footnotetext{
${ }^{1}$ K. S. H. Lee and C. H. Papas, J. Math. Phys. 5, 1668 (1964).

2 R. T. Compton, Jr., J. Math. Phys. 7, 2145 (1966).

${ }^{3}$ C. T. Tai, J. Math. Phys. 8, 646 (1967).

${ }_{4}^{4}$ J. M. Jauch and K. M. Watson, Phys. Rev. 74, 951 (1948).

5 C. Møller, The Theory of Relativity (Oxford U.P., London, 1952).

${ }^{6}$ J. L. Synge, Relativity: The Special Theory (North-Holland, Amsterdam, 1965).

${ }^{7}$ Equation (10) in Ref. 3 should contain a sum of two $\delta$ functions.

${ }^{8}$ C. T. Tai, IEEE Trans. Antennas Propagation 13, 322 (1965).
}

JOURNAL OF MATHEMATICAL PHYSICS VOLUME 11, NUMBER 11 NOVEMBER 1970

\title{
Expansion of an $n$-Point Function as a Sum of Commutators*
}

DON B. ZAGIER $\dagger$

Laboratory for Nuclear Science, Massachusetts Institute of Technology, Cambridge, Massachusetts

(Received 22 October 1969; Revised Manuscript Received 15 April 1970)

\begin{abstract}
We write the $n$-point function of currents as a sum over nested commutators, a form more suitable for certain current algebra calculations.
\end{abstract}

\section{INTRODUCTION AND RESULT}

In this paper we consider the $n$-point function, defined as

$$
\begin{aligned}
T\left(q_{1}, \cdots, q_{n-1}\right)_{\mu_{1} \cdots \mu_{n}} & \left.=\iint_{\times\langle 0|} \cdots \int^{4} d^{4} x_{1} \cdots d^{4} x_{n-1} e^{-i q_{1} x_{1}-\cdots-i q_{n-1} x_{n-1}}, \cdots j_{\mu_{n-1}-1}\left(x_{n-1}\right) j_{\mu_{n}}(0)\right)|0\rangle,
\end{aligned}
$$

where $T\left(j_{1} \cdots j_{n}\right)$ is the product of the $n$ current operators $j_{1}, \cdots, j_{n}$, in the order of the time components of their points of evaluation:

$$
\begin{aligned}
T\left(j_{1}\left(x_{1}\right) \cdots j_{n}\left(x_{n}\right)\right) & \\
=\sum_{\pi \in S_{n}} \theta\left(x_{\pi(1)}^{0}-\right. & \left.x_{\pi(2)}^{0}\right) \cdots \theta\left(x_{\pi(n-1)}^{0}-x_{\pi(n)}^{0}\right) \\
& \times j_{\pi(1)}\left(x_{\pi(1)}\right) \cdots j_{\pi(n)}\left(x_{\pi(n)}\right),
\end{aligned}
$$

where the sum is over all permutations $\pi$ in $S_{n}$, the symmetric group of order $n$. The function $\theta(t)$ is the usual step function. In Eq. (1) we only integrate over $n-1$ space variables because we are using the translation invariance of the theory to work in a coordinate system where $x_{n}=0$ and $q_{1}+\cdots+q_{n}=0$.

It is a straightforward calculation, which is given below, to rewrite Eq. (1) as a linear combination of products of $n$ current operators (or, rather, of their Fourier transforms in momentum space) not involving the step functions $\theta$. However, current algebra treats only commutators of operators rather than arbitrary products, so that it is desirable to express the $n$-point function as a linear combination of commutators of operators. 